\title{
Comment: childhood leukaemia and power lines - the Geocap study: is proximity an appropriate MF exposure surrogate?
}

\author{
M Bonnet-Belfais ${ }^{*}, 1, \mathrm{~J} \mathrm{Lambrozo}^{1}$ and A Aurengo ${ }^{2}$ \\ ${ }^{1}$ Medical Studies Department, EDF SA, Levallois-Perret, Paris, France and ${ }^{2}$ Nuclear Medicine Department, Hôpital \\ Pitie-Salpetriere, Paris, France
}

Sir,

For nearly four decades, the study of potential links between childhood leukaemia and exposure to extremely low frequency magnetic fields (MFs) has been the subject of numerous investigations worldwide (Sienkiewicz et al, 2010).

One of the essential difficulties in addressing the link between leukaemia and exposure to MFs is the complexity of the MF exposure assessment, and particularly its retrospective nature.

The Draper study (Draper et al, 2005) illustrated the limits of the distance to power lines as a surrogate of MF exposure, as an association with childhood leukaemia was observed up to $600 \mathrm{~m}$ from high-voltage (HV) and very high-voltage (VHV) lines (at this distance, MF exposure from a power line is not distinct from the background). Maslanyj et al (2009) have shown that 'exposure misclassification render the findings from studies that rely on distance alone uninterpretable'.

The Geocap study (Sermage-Faure et al, 2013), however, comes within the framework of the Draper study by using a quite comparable surrogate. Despite the care that the authors have taken for reconstituting the subjects' distance from power lines, this surrogate involves inaccuracies, leading to MF exposure classification errors.

The 32,779 subjects (2779 cases and 30000 controls) of the Geocap study are automatically placed in relation to the HV and VHV lines network at their date of inclusion in the study, by geocoding their postal addresses. 'Depending on whether the databases enabled location of the home directly or by extrapolation from the nearest or more distant neighbours,' the precision of 'automatic' postal address geocoding varies from $20 \mathrm{~m}$ to $>500 \mathrm{~m}$ (Table 1 in Sermage-Faure et al, 2013). Only 70\% of the cases and $77.2 \%$ of controls have a postal address geocoded with an uncertainty of $20 \mathrm{~m}$, and $>19 \%$ of cases and nearly $15 \%$ of controls have it geocoded with an uncertainty of at least $300 \mathrm{~m}$. Table 5 of the article (Sermage-Faure et al, 2013) provides some insight: among the 1258 subjects - 92 cases and 1166 controls - considered as living within $200 \mathrm{~m}$ of a line, only 68 cases and 863 controls have a geocoded address with an uncertainty of $20 \mathrm{~m}$. In addition, uncertainty in geocoding of cases is greater than that of controls.

Acknowledging the weakness of this automatic location, the authors completed their study with a further pinpointing using photographic views of the residential building from several sources (Streetview and Geoportail databases, French cadaster) for the 1258 subjects considered as living within $200 \mathrm{~m}$ of a line. This more accurate photo geocoding was obtained for 72 cases and 797 controls - that is, $<3 \%$ of the subjects in the study, but almost $70 \%$ of those considered as living within $200 \mathrm{~m}$ of a line. In particular, for the subjects close to VHV lines, photo geocoding is available for $>90 \%$ of the cases (30 among 33) and $67 \%$ of the controls (284 among 424). This photo geocoding resulted in a change from one distance class to another for a large proportion of the subjects living within $200 \mathrm{~m}$ of a line, notably for the cases closest to VHV lines, as among the nine cases classified by automatic distance within $50 \mathrm{~m}$ from a VHV power line only four were confirmed in this distance class by photo geocoding.

This demonstrates the instability of the subjects' classification regarding their residential proximity to power lines, which is strongly dependent on the geocoding used. It confirms that the use of distance as a surrogate for MF exposure is inappropriate and should be discouraged in favour of personal exposure - the only exposure measure relevant in terms of public health.

These uncertainties have not been included in the main analysis. The main result, presented in the abstract, is not based on the most accurate location, but on the 9 cases and 60 controls supposed to live within $50 \mathrm{~m}$ of a VHV line according to the automatic geocoding. The result is a non-significant increased risk of leukaemia in children less than 15 years of age: $\mathrm{OR}=1.7(0.9-3.6)$. 
These uncertainties concerning power line distance lead to a very rough estimate of the child exposure to the MF produced by a power line (which is the subject of the study), as MF decreases in inverse proportion to the square of the distance. Moreover, this estimate takes into account neither the effective residence duration in the vicinity of the power lines nor other sources of exposure - at home or in other places such as at nursery or at school - nor other potential confounding factors.

\section{REFERENCES}

Draper G, Vincent T, Kroll M, Swanson J (2005) Childhood cancer in relation to distance from high voltage power lines in England and Wales: a case-control study. BMJ 330: 1290.
Maslanyj M, Simpson J, Roman E, Schüz J (2009) Power frequency magnetic fields and risk of childhood leukemia: misclassification of exposure from the use of the 'distance from power line' exposure surrogate. Bioelectromagnetics 30: 183-188.

Sermage-Faure C, Demoury C, Rudant J, Goujon-Bellec S, Guyot-Goubin A, Deschamps F, Hemon D, Clavel J (2013) Childhood leukaemia close to high-voltage power lines-the Geocap study, 2002-2007. Br J Cancer 108: 1899-1906.

Sienkiewicz Z, Schüz J, Poulsen AH, Cardis E (2010) Risk analysis of human exposure to electromagnetic fields. European Health Risk Assessment Network on Electromagnetic Fields Exposure (EFHRAN). Available from http://efhran.polimi.it/docs/EFHRAN_D2_final.pdf.

(c) (1) (2) This work is licensed under the Creative Commons cc) Attribution-NonCommercial-Share Alike 3.0 Unported License. To view a copy of this license, visit http://creativecommons. org/licenses/by-nc-sa/3.0/

\section{Reply: Comment on 'Childhood leukaemia close to high-voltage power lines - the Geocap study, 2002-2007' - Is proximity an appropriate MF exposure surrogate?}

J Clavel ${ }^{\star, 1,2,3}$, C Sermage-Faure ${ }^{1,2}$, C Demoury ${ }^{1,2}$, J Rudant ${ }^{1,2,3}$, S Goujon-Bellec ${ }^{1,2,3}$, A Guyot-Goubin ${ }^{1,2,3}$, F Deschamps ${ }^{4}$ and D Hémon ${ }^{1,2}$

${ }^{1}$ Inserm U1018, Center for Research in Epidemiology and Population Health (CESP) Equipe 6, Environmental Epidemiology of Cancer Team, 16 Av. Paul Vaillant-Couturier, F-94807 Villejuif, France; '2University Paris-Sud, UMRS 1018, F-94807 Villejuif, France; ${ }^{3}$ French National Registry of Childhood Hematological Malignancies (NRCH), Villejuif, France and ${ }^{4}$ Réseau de Transport d'Electricité (RTE), Coeur Défense, 110 Esplanade du Général de Gaulle, 92030 La Defense, France

Sir,

We would like to thank Bonnet-Belfais et al (2013) for their interest in our work. We fully agree that addressing the possible link between childhood acute leukaemia (AL) and exposure to magnetic fields (MF) is a difficult task. In our article, we analysed the relationship between $\mathrm{AL}$ and close proximity to high-voltage overhead lines (HVOL), not exposure to MF. The methods, results, discussion and conclusions did not deviate from that objective. We did not use the distance from HVOL as a proxy of ELF-MF exposure, but as a marker of a subpopulation of French children who, on average, are expected to be exposed more often to higher levels of ELF-MF because they live close to HVOL.

Bonnet-Belfais et al (2013) considered the coordinates based on photographic views as an accurate gold standard and contrasted them with the coordinates obtained by the main geocoding

${ }^{*}$ Correspondence: Dr J Clavel; E-mail: Jacqueline.Clavel@inserm.fr

Published online 15 August 2013

(C) 2013 Cancer Research UK. All rights reserved 0007-0920/13

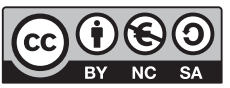

BPEN 0 